\title{
LETTER \\ A Simple but Effective Congestion Control Scheme for Safety-Related Events in VANET
}

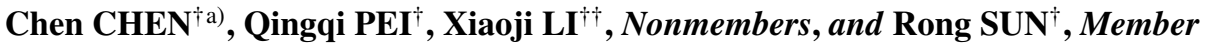

\begin{abstract}
SUMMARY In this letter, a Simple but Effective Congestion Control scheme (SECC) in VANET has been proposed to guarantee the successful transmissions for safety-related nodes. The strategy derive a Maximum Beacon Load Activity Indicator (MBLAI) to restrain the neighboring general periodical beacon load for the investigated safety-related "observation nodes", i.e., the nodes associated with some emergent events. This mechanism actually reserves some bandwidth for the safety-related nodes to make them have higher priorities than periodical beacons to access channel. Different from the static congestion control scheme in IEEE802.11p, this strategy could provide dynamic control strength for congestion according to tolerant packets drop ratio for different applications.

key words: vehicular ad hoc networks, congestion control, safety-related, IEEE802.11p
\end{abstract}

\section{Introduction}

VANET is being developed for applications including mainly safety-related scenarios, such as Cooperative forward Collision Warning (CCW), traffic signal violation warning, lane change warning and some information applications. The main way of information dissemination for safety-related applications in VANET relies on periodical beacon broadcasting. For safety-related applications, the correctness and up-to-datedness of the messages greatly depend on the successful emergent broadcast receive ratio and periodical beacons frequency. However, it has been observed that the performance of the broadcast based safety applications drops down seriously in dense scenarios, such as channel congestion due to heavy beacon load resulting in strong interferences at receivers. To our best knowledge, at present, the popular congestion control strategy proposed in VANET is to prevent any message, except from the highest priority, to be transmitted if the measured channel occupancy is larger than 50\% [1]. This strategy, in fact, is a bandwidth reservation method for emergent events by restraining the periodic beacons load. Although this scheme sounds reasonable, there are no detailed implementation methods and related measurement ways published to make it practical.

\footnotetext{
Manuscript received April 5, 2012.

${ }^{\dagger}$ The authors are with the Faculty of the School of Telecommunication, the Xidian University and also with the State Key Laboratory of Integrated Service Networks, Xidian University, P.R. China.

${ }^{\dagger}$ The author is with the Faculty of the School of Electronic Engineering, the Guilin University of Electronic Technology, P.R. China.
}

a)E-mail: cc2000@mail.xidian.edu.cn

DOI: 10.1587/transinf.E95.D.2548

\section{Proposed Congestion Control Scheme}

In our work, congestion control in VANET tends to avoid more severe jam before the performance of safety-related applications begins to degrade greatly. The objective is obtained by limiting the beacons' activity within a Maximum Beacon Load Activity Indicator (MBLAI). We will first introduce some assumptions and then describe our model in detail.

\subsection{Assumptions and Model description}

As we have indicated in Sect. 1, congestion control in VANET with dynamic network parameters is really a NPHard problem and need to be relaxed to evaluate the performance in different scenarios. Therefore, we convert the dynamically determined problem to a relatively static one with following assumptions:

Assumption 1: The SINR threshold for successfully decoding the received signal is fixed at $S I N R_{t h}$ for all vehicles; Assumption 2: The transmitting power for each burst is fixed at $P_{t}$ for all vehicles.

Assumption 3: The distances between sources and destinations can be readily obtained by the Receive Signal Strength Indicator (RSSI) under LOS (Line Of Sight) environment or hybrid TOA/AOA [2] algorithms under NLOS (Non Line Of Sight) circumstance.

With the above assumptions, we can formulate the relation of transmitting power at receiver and at transmitter as:

$$
P T_{i} \cdot G_{i, j}=P R_{j}
$$

where $P T_{i}$ and $P R_{j}$ are the transmitting power at transmitter $i$ and received signal strength at receiver $j$ respectively. $G_{i, j}$ denotes the experienced channel gain from $i$ to $j$ and is modeled as:

$$
G_{i, j}=\frac{K}{d_{i, j}^{n}}
$$

where $K$ is a constant and $d_{i, j}$ is the distance between $i$ and $j$ with $n$ as the path loss factor. For a given signal decoding threshold $S I N R_{t h}$, the allowed noise power at $j$ is

$$
P N_{j}=\frac{P R_{j}}{S I N R_{t h}}
$$

Considering the thermal noise and interferences from other 
ongoing links, the total noise power at $j$ can also be expressed as:

$$
P N_{j}=P T H_{j}+\sum_{m}\left\{G_{m, j} \cdot P T_{m}: j \in C S\left(P_{m}\right)\right\}
$$

where is the thermal noise around $j$ and $C S\left(P_{m}\right)$ denotes the carrier-sense range of $m$ under the transmitting power $P_{m}$. In the NS2 simulator, a default carrier sensing range of 2.2 times the transmission range is suggested for IEEE 802.11 series protocols. This static value is optimal in many network scenarios [3] and we also take this setting.

Now, with Eq. (1-4), for the transmitter $i$ and receiver $j$, we have

$$
P N_{j}^{*}=\frac{P T_{i} \cdot G_{i, j}}{S I N R_{t h}}=\frac{P T_{i} \cdot K}{S I N R_{t h} \cdot D_{j}^{n}}
$$

Where $D_{j}$ denotes the maximum transmission range of node $j$, which is set the same to all nodes in overall network. The definition of $P N_{j}^{*}$ intends to derive a lower bound for the allowable receiving power involving interferences at $j$, which could be later taken as the permitted Maximum Beacon Load Activity Indicator (MBLAI) around $j$. By Assumption 1 and 2 , we can obtain the exact value of maximum allowed noise at $j$, i.e., $P N_{j}$. In other words, the congestion could be controlled if the total interferences at $j$ satisfy the following inequality:

$$
P N_{j} \leq P N_{j}^{*}
$$

The definition of $P N_{j}^{*}$ in our proposed MBLAI, in fact, set the allowable beacon load around the intent receiver $j$.

\subsection{Description of SECC Control Procedure}

To make our SECC scheme practical, we introduce a parameter AP (Approaching Percentage), which indicates the distance between current beacon load and MBLAI. Considering the high dynamic property of VANET due to mobility, a real time control scheme is computation consuming with a great number of overheads, which still could not catch the ongoing traffic and topology changing. Therefore, we execute SECC with interval $T$ seconds, and make SECC active with $A P$ reserved bandwidth in advance. When congestion detected by SECC will occur, we turn to the duration field in beacon header, which is plotted in Fig. 1, to restrain the neighboring traffics. With NAV (Network Allocation Vector) parameter in duration fields, the Onode, i.e. Observation node, could broadcast a larger NAV value to hinder part of the traffics generated on neighbors. The detailed working procedure of our proposed SECC is coded in Fig. 2. $d$ and $p$ indicates the duration for restraining neighbors and proportion of neighbors selected to be controlled, respectively. Function order sort the neighbors by their channel gains in ascending order. Function select choose front $\% p$ neighbors from ordered list to restrain. Function setNAV set the NAV fields of selected list to $d$ period.

Octets: \begin{tabular}{c|c|c|c|c|c|c|c|}
2 & 2 & 6 & 6 & 6 & 2 & $0-2312$ & 4 \\
\hline $\begin{array}{c}\text { Frame } \\
\text { Control }\end{array}$ & Duration & DA & SA & BSSID & $\begin{array}{c}\text { Sequence } \\
\text { Control }\end{array}$ & $\begin{array}{c}\text { Beacon } \\
\text { Body }\end{array}$ & CRC \\
\hline
\end{tabular}

Fig. 1 Beacon header format.

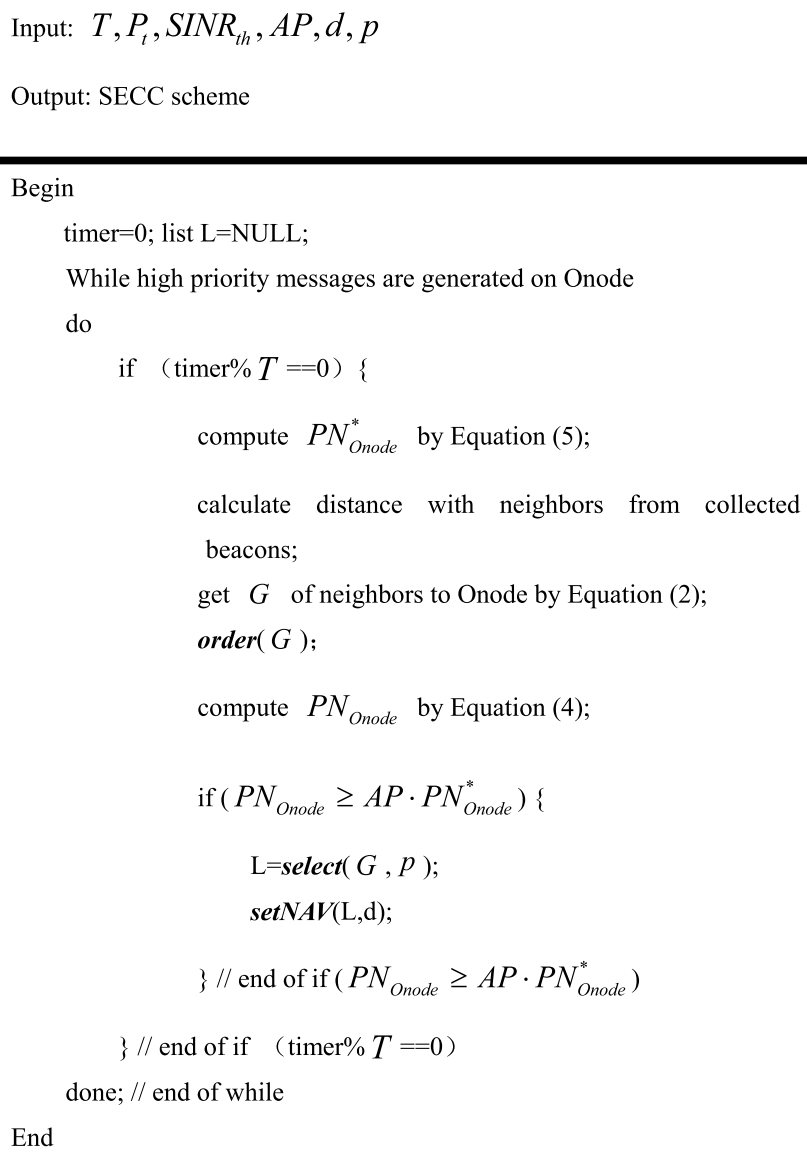

Fig. 2 Pseudocode of SECC.

\section{Performance Evaluation and Numerical Results}

In this section, we will evaluate the performance of our proposed scheme by Vanetmobisim and NS2.The general simulation parameters are listed in Table 1.

The Queue model used in our simulation is just DropTail strategy due to the simplified setting for routing scheme,i.e., DumbAgent, which implies that no routing is required.

The Simulation topology is plotted in Fig. 3 in a $1000 \mathrm{~m} \times 1000 \mathrm{~m}$ field where 50 vehicles are uniformly distributed and moving following the IDM-IM mobility model. The intersections are randomly generated by applying a Voronoi tessellation to a set of randomly distributed points. The Onode is designated as node 0 . The other parameters for IDM-IM model are listed in Table 2. Based on the introduction and analysis in Sect. 2, we could launch the congestion control strategy according to Fig. 2 on a node, i.e. 
Table 1 Simulation common parameters setting.

\begin{tabular}{cccc}
\hline Parameters & values & Parameters & values \\
\hline Propagation model & TwoRayGround & Mac & 802.1 lextent \\
$\mathrm{T} / \mathrm{s}$ & 10 & $\mathrm{~d} / \mathrm{s}$ & 5 \\
$\mathrm{p}$ & $20 \%$ & Number of vehicles & 50 \\
Packet size $/ \mathrm{B}$ & 250 & Simulation period $/ \mathrm{s}$ & 300 \\
Simulation field $/ \mathrm{m} \times \mathrm{m}$ & $1000 \times 1000$ & Path loss factor & 2.2 \\
SINR $_{\mathrm{th}} / \mathrm{dBm}$ & -90 & $\mathrm{P}_{\mathrm{t}} / \mathrm{mW}$ & 0.3754 \\
transmission range $/ \mathrm{m}$ & 300 & Frequency $/ \mathrm{Hz}$ & $5.9 \mathrm{e}+9$ \\
\hline
\end{tabular}

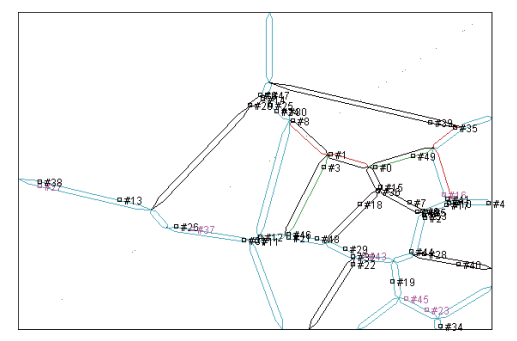

Fig. 3 Simulation topology.

Table 2 scenario setting.

\begin{tabular}{llll}
\hline \multicolumn{1}{c}{ Parameters } & values & Parameters & values \\
\hline Lane number & 2 & Traffic light & 2 \\
Min.Stay(s) & 0.5 & Max. Stay(s) & 10 \\
Mobility model & IDM-IM & Traffic rate(packets/s) & 10 \\
\hline
\end{tabular}

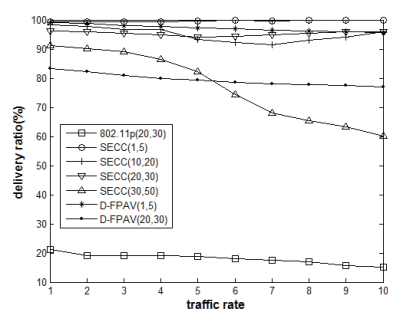

Fig. 4 delivery ratio vs. traffic rates.

the observation node Onode, by limiting or restraining the neighboring traffic flows before beacon load activity is approaching the MBLAI $P N_{\text {Onode }}^{*}$.

Figure 4 shows the delivery ratio performance comparison between IEEE802.11p broadcasting scheme, DFPAV [4] (Distributed Fair Power Adjustment for Vehicular networks) and our proposed SECC model with $A P=50 \%$, which means our SECC model will work when current beacon load activity on Onode approaching $50 \%$ of the MBLAI $P N_{\text {Onode }}^{*}$. The symbol SECC (x,y) expresses that our SECC model runs with a minimum velocity $\mathrm{x}$ and a maximum velocity y respectively for all nodes. The traffic rate is represented by the number of packets sent per second, which in fact is taken as the beacon frequency in our simulations. As shown in Fig. 4, the delivery ratios of our SECC model are always higher than that of IEEE802.11p with different

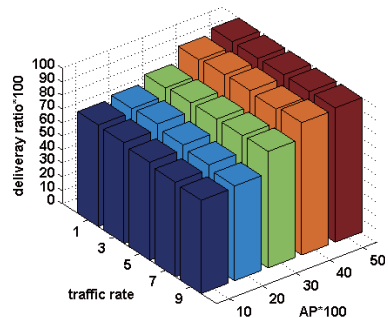

Fig. 5 delivery ratio vs. traffic rates and $A P$.

velocity settings. The delivery ratio of IEEE802.11p is still less than $20 \%$ during the overall simulation period when the minimum and maximum velocity are 20 and 30 respectively. Further, it has a lowest value of only $15.1 \%$ when traffic rate is 10 packets/s. However, with $A P=50 \%$, our SECC model could greatly improve the number of successfully delivered packets on Onode and restrain the unnecessary transmitting attempts that would result in large number of collisions and energy consuming when network is approaching congestion. The performance of SECC model is relatively steady with traffic rates change and the fluctuation begin to occur only when minimum velocity is larger than approximate $30 \mathrm{~m} / \mathrm{s}$. The reason is that the high velocity makes network more dynamic and cause our SECC model, which is based on the perception and measurement of the number of surrounding active nodes and the distances between Onode and its neighbors, not competent for such dramatic topology change. For D-FPAV, because it is a power control based distributed congestion control algorithm that introduces game theory based Max-Min strategy, it also has a better performance when velocity is low. However, when mobility level increase, DFPAV shows worse delivery ratio than SECC due to its larger convergence time that make D-FPAV cannot catch the topology changing.

The delivery ratio performance on different values of $A P$ has been evaluated as shown in Fig. 5. The velocity is set to uniform $(10,20)$ meaning that the driving speed is uniformly chosen from minimum velocity $10 \mathrm{~m} / \mathrm{s}$ to maximum velocity $20 \mathrm{~m} / \mathrm{s}$. The results show that the delivery ratio decreases with $A P$ falling but there is still approximate $70 \%$ packets successfully received by Onode. The performance falling when $A P$ is small is due to the underestimation of the current beacon load and not reserving enough delay and bandwidth for congestion control mechanism working. In our simulation, it is only taken $0.5 \mathrm{~s}$ to make $A P$ from $10 \%$ to zero, which may not meet for the delay requirement for broadcasts dissemination to update active neighbors and congestion level estimation.

\section{Conclusions}

We have proposed a congestion control strategy to alleviate the severe packets collisions and traffic jam in VANET. This strategy could provide flexible control dynamics to vehicles that are associated with safety-related events and eager for higher priorities than general periodical beacons to dissem- 
inate emergent warnings.

\section{Acknowledgements}

This work has been supported by the National Natural Science Foundation of China (61201133; 61172055;60832005; U0835004; 61072067), the Postdoctoral Science Foundation of China (20100481323), the Program for New Century Excellent Talents (NCET-11-0691), the "111 Project" of China (B08038), and the Foundation of Guangxi Key Lab of Wireless Wideband Communication \& Signal Processing (11105).

\section{References}

[1] H. Hartenstein and K. Laberteaux, VANET Vehicular Applications and Inter-Networking Technologies, Wiley Online Library, 2009.

[2] S. Venkatraman and J. Caffery, Jr., "Hybrid TOA/AOA techniques for mobile location in non-line-of-sight environments," IEEE Wireless Communications and Networking Conference, pp.274-278, Atlanta, Georgia, USA, March 2004.

[3] J. Deng, B. Liang, and P.K. Varshney, "Tuning the carrier sensing range of IEEE 802.11 MAC," IEEE Global Telecommunications Conference, pp.2987-2991, Dallas, Texas, USA, Nov. 2004.

[4] M. Torrent-Moreno, P. Santi, and H. Hartenstein, "Distributed fair transmit power adjustment for vehicular ad hoc networks," 3rd Annual IEEE Communications Society on Sensor and Ad Hoc Communications and Networks, pp.479-488, Reston, VA, USA, Sept. 2006. 